\title{
Critical behavior of drops in linear flows: I. Phenomenological theory for drop dynamics near critical stationary states
}

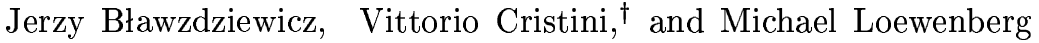 \\ Department of Chemical Engineering, Yale University, New Haven, Connecticut 06520-8286
}

\begin{abstract}
The dynamics of viscous drops in linear creeping flows are investigated near the critical flow strength at which stationary drop shapes cease to exist. It is shown that the near-critical drop behavior is dominated by a single slow mode that evolves on the time scale diverging at the critical point with the exponent $\frac{1}{2}$. Our theory, based on the assumption that the system undergoes a saddle-node bifurcation at the critical state, has been verified numerically for drops in shear flow and axisymmetric straining flow. A good agreement is obtained between the analytical predictions and numerical results. An important application of our theory for an accurate determination of critical parameter values is discussed.
\end{abstract}

\section{INTRODUCTION}

Emulsion properties, such as rheology, depend strongly on the drop size distribution. Thus, it is important to understand and predict drop breakup in creeping flows, and there has been much research on this topic (as reviewed by Stone $^{1}$ ). Recent studies include drop breakup in stationary ${ }^{2-5}$, and time-dependent ${ }^{6-11}$ flows. Some of the research has been focused on the criteria for breakup and some on the drop fragments produced by individual breakup events.

In stationary flows, criteria for breakup can be expressed in terms of a critical capillary number, i.e., the (dimensionless) flow strength above which no stationary drop shapes exist. The critical capillary number depends on the viscosity ratio of the drop, and the form of the flow. In transient flows, criteria for breakup depend on the flow strength as well as the flow history and the initial drop shape.

Recently, Navot ${ }^{12}$ explored drop dynamics in a stationary axisymmetric straining flow under conditions close to the critical capillary number. Using numerical simulations, Navot demonstrated that the time scale for drop evolution diverges at the critical capillary number with the exponent $\frac{1}{2}$, and the stationary drop length exhibits a non-analytical, square-root behavior in the near-critical regime. The main features of the near-critical drop behavior were qualitatively explained using a simple oneparameter model with the drop shape parameterized by the drop length.

In a recent presentation ${ }^{13}$, we reported similar observations and described a systematic analysis of drop dynamics under near-critical conditions. We showed that in the near-critical regime, a one-parameter description of drop-shape evolution holds because of the separation of time scales. The slow time scale corresponds to a single critical mode that becomes unstable at the critical capillary number. The remaining stable modes evolve on a fast time scale; thus, at long times, they adiabatically follow the evolution of the slow critical mode. The details of this analysis are described in the present paper.

\section{DEFORMABLE DROPS IN LINEAR FLOWS}

\section{A Velocity field}

We consider the evolution of a viscous drop of volume $\frac{4}{3} \pi a^{3}$ immersed in an unbounded fluid undergoing stationary Stokes flow. The viscosity of the continuous phase is $\mu_{1}$ and the drop phase is $\mu_{2}=\eta^{-1} \mu_{1}$. The drop interface has constant interfacial tension $\sigma$.

A linear external velocity field is assumed,

$$
\mathbf{v}_{0}=\dot{\gamma} \mathbf{D} \cdot \mathbf{r},
$$

where $\mathbf{r}$ is the position, $\dot{\gamma}$ is the magnitude of the flow, and $\mathbf{D}$ is the normalized velocity-gradient tensor (which is asymmetric and traceless).

The fluid velocity $\mathbf{v}$ and pressure $p$ in phase $(i)$ satisfy the Stokes equations,

$$
\mu_{i} \nabla^{2} \mathbf{v}-\nabla p=0, \quad \nabla \cdot \mathbf{v}=0
$$

At the drop interface $S$, velocity field and tangential stresses are continuous, and the jump of normal stresses across $S$ is equal to the capillary pressure,

$$
[\mathbf{n} \cdot \boldsymbol{\tau} \cdot \mathbf{n}]_{S}=2 C \sigma \mathbf{n},
$$

where $\boldsymbol{\tau}$ is the stress tensor, $\mathbf{n}$ is the outwards normal vector, and $C$ is the local mean curvature. At infinity, velocity field $\mathbf{v}$ satisfies the boundary condition

$$
\mathbf{v}=\mathbf{v}_{0} .
$$

According to Eqs. (1)-(4), drop evolution depends on the viscosity ratio $\eta$, the capillary parameter

$$
\kappa=\frac{\sigma}{a \mu_{1} \dot{\gamma}}
$$


(the inverse capillary number), and four dimensionless parameters characterizing the form of the external flow. In the present paper, we examine the dependence of drop evolution on $\kappa$, for given $\eta$ and the form of the external flow.

\section{B Evolution of the drop shape}

To characterize the instantaneous drop shape, we introduce an array of shape parameters f. (An example of such an array is the set of expansion coefficients of the radial coordinate of the interface into spherical harmonics ${ }^{14,15}$.) The general arguments presented in this paper, however, are independent of the particular choice of $\mathbf{f}$.

The evolution of the drop shape results from the motion of the interface with the fluid velocity $\mathbf{v}$. The velocity field is nonlinear in $\mathbf{f}$, but for a given drop shape the boundary-value problem (2)-(4) is linear. Thus, $\mathbf{v}$ can be decomposed into convective and relaxation parts,

$$
\mathbf{v}=a \dot{\gamma} \mathbf{v}_{\dot{\gamma}}+\mu_{1}^{-1} \sigma \mathbf{v}_{\sigma}
$$

where the first term represents the velocity field produced by an incident flow (1) in the absence of capillary forces, and the second term is the capillary-driven fluid velocity in the absence of an incident flow.

Decomposition (6) implies the structure of the evolution equation for the array of shape parameters. In dimensional variables with time normalized by $\dot{\gamma}^{-1}$ and distances normalized by $a$, the evolution equation has the form

$$
\frac{\mathrm{d} \mathbf{f}}{\mathrm{d} t}=\mathbf{G}(\mathbf{f})-\kappa \mathbf{H}(\mathbf{f})
$$

where the function $\mathbf{G}(\mathbf{f})$ results from the convective velocity contribution $\mathbf{v}_{\mathrm{e}}$, and the function $\mathbf{H}(\mathbf{f})$ is associated with the capillary-driven velocity $\mathbf{v}_{\mathrm{c}}$.

Explicit expressions for the nonlinear functions $\mathbf{G}$ and $\mathbf{H}$ are known only in the regime of small drop deformations (see Refs. 14-16). Outside this regime, $\mathbf{G}$ and $\mathbf{H}$ can be evaluated numerically. In the present work, important features of drop behavior near critical stationary states are derived from the drop-shape evolution equation in its most general form (7), supplemented by some general assumptions.

\section{NEAR-CRITICAL DROP BEHAVIOR}

\section{A Critical capillary parameter}

According to experimental and numerical results ${ }^{1}$, for a given viscosity ratio and type of flow, there is a range of capillary numbers for which stable stationary drop shapes
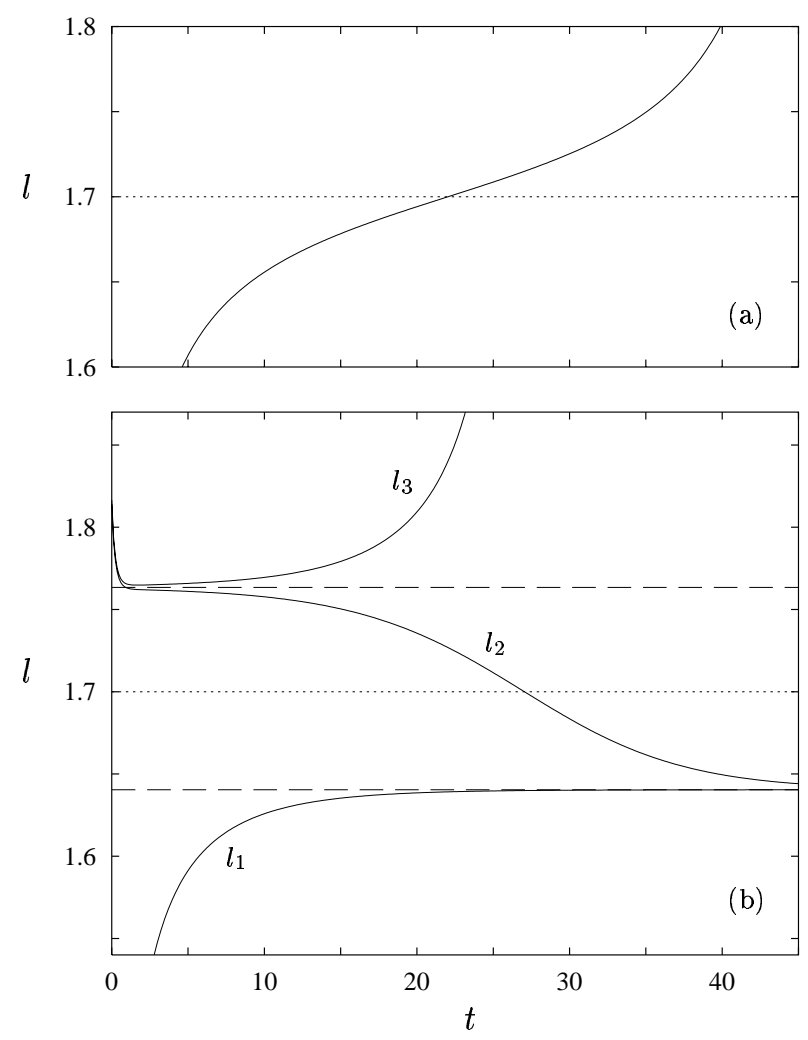

FIG. 1: Drop length versus time for initially spheroidal drops $(\eta=1)$ in axisymmetric strain; $\kappa / \kappa_{\mathrm{C}}=0.998(a)$ and $\kappa / \kappa_{\mathrm{C}}=1.004(b)$, where $\kappa_{\mathrm{C}}$ is given by Eq. (48). Drop length evolution with different initial conditions (solid lines); stationary drop lengths (dashed lines); critical length (dotted line).

exist. Typically, stable stationary solutions form a continuous branch $\mathbf{f}_{\mathrm{st}}=\mathbf{f}_{\mathrm{st}}(\kappa)$ that exists for

$$
\kappa>\kappa_{\mathrm{C}}
$$

where $\kappa_{\mathrm{C}}$ is the critical value of the capillary parameter. Herein, we consider this case.

\section{B Drop evolution in the near-critical regime}

Some general features of drop behavior in the nearcritical regime are illustrated in Figs. 1-3, where evolution of the drop length (normalized by the drop diameter $2 a$ ) is shown for a drop with $\eta=1$ in axisymmetric straining flow

$$
\mathbf{v}_{0}=\dot{\gamma}\left(z \hat{\mathbf{e}}_{z}-\frac{1}{2} \rho \hat{\mathbf{e}}_{\rho}\right) .
$$

Here $(\rho, z)$ are cylindrical coordinates, and $\hat{\mathbf{e}}_{\rho}, \hat{\mathbf{e}}_{z}$ are the corresponding unit vectors. The numerical results presented in Figs. 1-3 were obtained using a boundary integral algorithm ${ }^{17}$.

In Fig. 1(a), drop-length history is shown for a subcritical value of the capillary parameter, $\kappa<\kappa_{\mathrm{C}}$. No 


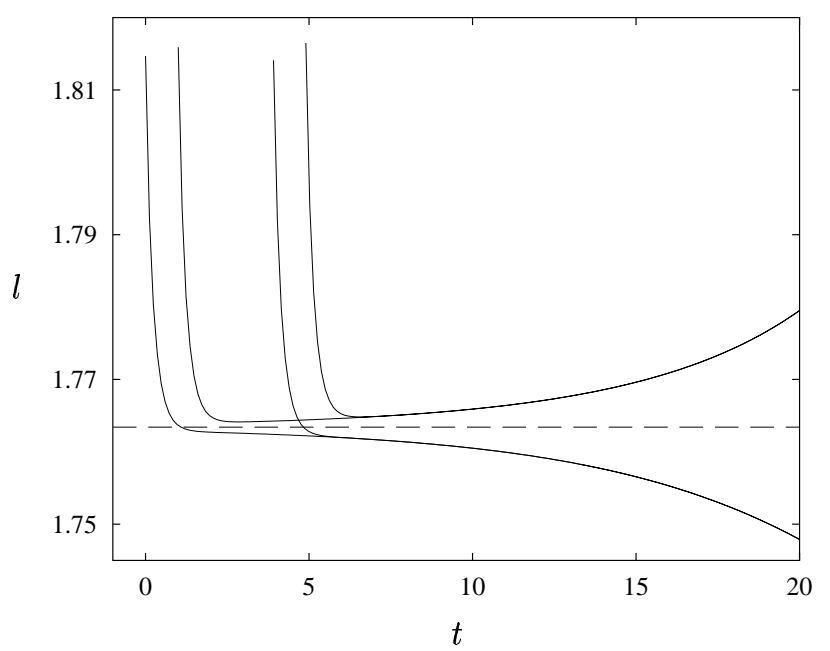

FIG. 2: Short-time evolution of drop length $l$ for initially spheroidal drops $(\eta=1)$ in axisymmetric strain, for $\kappa / \kappa_{\mathrm{C}}=$ 1.004. Time-dependent solutions with different initial conditions (solid lines); unstable stationary solution (dashed line).

stationary states exist in this case, and the length increases continuously (until the drop breaks). In contrast, two stationary drop shapes exist for supercritical values of capillary parameter $\kappa>\kappa_{\mathrm{C}}$, as shown in Fig. 1(b). The results indicate that the stationary shape with the smaller length, $l=l_{-}$, is stable, and the shape with the larger length, $l=l_{+}$, is unstable.

The results presented in Fig. 1(b) and the detail of the initial evolution shown in Fig. 2 indicate that the drop evolves on two distinct time scales in the near-critical regime. At short times, evolution occurs on the capillaryrelaxation time scale $\tau_{\kappa}=(\kappa \dot{\gamma})^{-1}$; afterwards, the drop evolves on much longer time scale.

In the long-time regime, drop evolution is insensitive to the details of the initial conditions, as illustrated in Figs. 1 and 2. For $\kappa<\kappa_{\mathrm{C}}$ the long-time portion of the trajectory corresponds to a unique sequence of states. For $\kappa>\kappa_{\mathrm{C}}$, after the long-time regime has been achieved, the drop evolves along one of the three trajectories $l=$ $l_{i}(t)$, where

$$
l_{1}(t)<l_{-}, \quad l_{-}<l_{2}(t)<l_{+}, \quad l_{3}(t)>l_{+}
$$

(as labeled in Fig. 1).

This behavior is further illustrated in Fig. 3, where the relation between drop length $l$ and its time derivative $l$ is shown for a subcritical, critical, and supercritical value of the capillary parameter. For $\kappa \geq \kappa_{\mathrm{C}}$, the three portions of the continuous curve $i(l)$ that correspond to different long-time trajectories $l=l_{i}(t)$ were obtained using different initial conditions (a sphere for $i=1$, and elongated spheroids with different lengths for $i=2$ and $i=3$ ). As indicated by the dashed lines corresponding to the evolution on the fast time scale, the details of the initial conditions become irrelevant at long times.

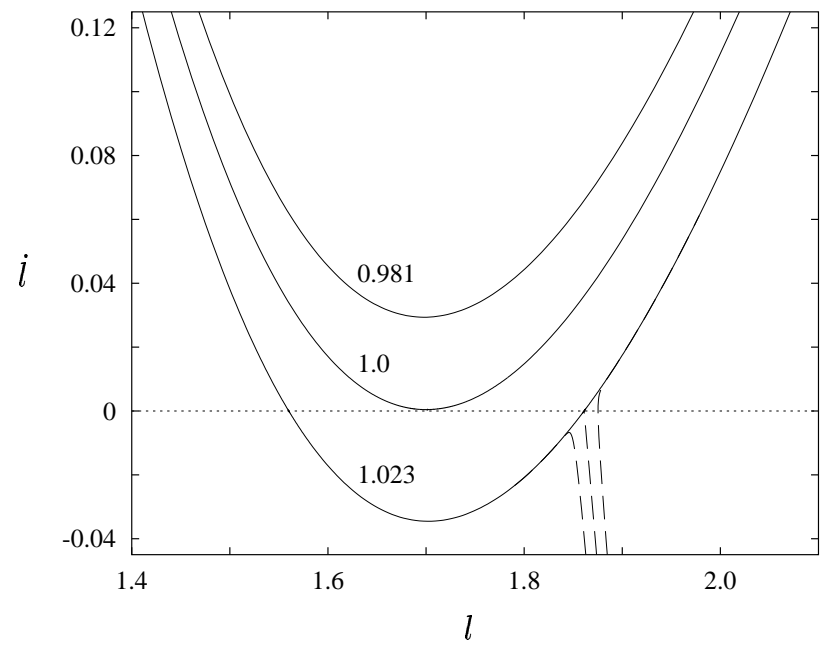

FIG. 3: Rate of change of drop length $i$ versus drop length $l$, for viscous drops $(\eta=1)$ in axisymmetric strain. Long-time evolution (solid lines); short-time evolution with different initial conditions (dashed lines). Values of $\kappa / \kappa_{\mathrm{C}}$ as labeled.

The results presented in Figs. 1-3 suggest that at $\kappa=\kappa_{\mathrm{C}}$ a saddle-node bifurcation occurs, and the critical stationary drop shape corresponds to a turning-point singularity. ${ }^{18}$ In the following sections, we develop an appropriate description.

\section{SLOW MODE DESCRIPTION}

\section{A Expansion of the evolution equation}

To characterize drop behavior in the near-critical regime, Eq. (7) is expanded in a Taylor series around the critical stationary shape

$$
\mathbf{f}_{\mathrm{C}}=\mathbf{f}_{\mathrm{st}}\left(\kappa_{\mathrm{C}}\right) .
$$

A regular expansion in

$$
\delta \mathbf{f}=\mathbf{f}-\mathbf{f}_{\mathrm{C}}
$$

yields

$$
\begin{aligned}
\frac{\mathrm{d}}{\mathrm{d} t} \delta \mathbf{f}= & \mathbf{G}\left(\mathbf{f}_{\mathrm{C}}\right)-\kappa \mathbf{H}\left(\mathbf{f}_{\mathrm{C}}\right)+\left[\mathbf{G}^{(1)}\left(\mathbf{f}_{\mathrm{C}}\right)-\kappa \mathbf{H}^{(1)}\left(\mathbf{f}_{\mathrm{C}}\right)\right] \cdot \delta \mathbf{f} \\
& +\frac{1}{2}\left[\mathbf{G}^{(2)}\left(\mathbf{f}_{\mathrm{C}}\right)-\kappa \mathbf{H}^{(2)}\left(\mathbf{f}_{\mathrm{C}}\right)\right]: \delta \mathbf{f} \delta \mathbf{f}+\ldots,
\end{aligned}
$$

where $\mathbf{A}^{(i)}$ denotes the $i^{\text {th }}$ derivative of $\mathbf{A}$ with respect to $\mathbf{f}$.

The critical drop shape $\mathbf{f}_{\mathrm{C}}$ is stationary at $\kappa=\kappa_{\mathrm{C}}$, which yields

$$
\mathbf{G}\left(\mathbf{f}_{\mathrm{C}}\right)-\kappa_{\mathrm{C}} \mathbf{H}\left(\mathbf{f}_{\mathrm{C}}\right)=0 .
$$

For $\kappa>\kappa_{\mathrm{C}}$ there are stationary shapes $\mathbf{f}_{\mathrm{st}}(\kappa)$ that are stable to small perturbations, which indicates that all 
eigenvalues $\lambda_{i}$ of the matrix $\mathbf{G}^{(1)}\left(\mathbf{f}_{\mathrm{st}}\right)-\kappa \mathbf{H}^{(1)}\left(\mathbf{f}_{\mathrm{st}}\right)$ have negative real parts. Based on our numerical results, we assume that only a single mode becomes unstable at the critical state. Accordingly, at $\kappa=\kappa_{\mathrm{C}}$,

$$
\lambda_{1}=0 \text {, }
$$

and

$$
\operatorname{Re}\left(\lambda_{i}\right)<0, \quad i=2,3 \ldots,
$$

where

$$
\left[\mathbf{G}^{(1)}\left(\mathbf{f}_{\mathrm{C}}\right)-\kappa_{\mathrm{C}} \mathbf{H}^{(1)}\left(\mathbf{f}_{\mathrm{C}}\right)\right] \cdot \mathbf{g}_{i}=\lambda_{i} \mathbf{g}_{i},
$$

with eigenvectors $\mathbf{g}_{i}$ forming a nonorthogonal basis in the space of shape parameters.

Equations (13)-(17) imply that for $\kappa \approx \kappa_{\mathrm{C}}$ drop-shape perturbations are naturally decomposed as

$$
\delta \mathbf{f}=\delta \mathbf{f}_{\mathrm{s}}+\delta \mathbf{f}_{\mathrm{f}}
$$

into the slow mode

$$
\delta \mathbf{f}_{\mathrm{s}}=\delta f_{\mathrm{s}} \mathbf{g}_{1}
$$

and fast modes

$$
\delta \mathbf{f}_{\mathbf{f}}=\sum_{i=2}^{\infty} \delta f_{i} \mathbf{g}_{i} .
$$

The time scale for the evolution of the slow mode diverges for $\kappa \rightarrow \kappa_{\mathrm{C}}$ according to Eq. (15); in contrast, fast modes always evolve on the non-singular capillaryrelaxation time scale. This separation of the time scales results in a simplified drop dynamics in the near-critical regime: after an initial equilibration on the capillary time scale, fast modes follow the evolution of the slow mode in a quasistatic way, and can, thus, be eliminated from the evolution equations.

As shown in appendix A 1, the elimination of the fast modes yields a closed evolution equation for the amplitude of the slow mode. To the leading order we obtain

$$
\frac{\mathrm{d}}{\mathrm{d} t} \delta \bar{f}_{\mathrm{s}}=\epsilon\left(-s c_{0}+c_{2} \delta \bar{f}_{\mathrm{s}}^{2}\right)+\epsilon^{2}\left(s c_{1} \delta \bar{f}_{\mathrm{s}}+c_{3} \delta \bar{f}_{\mathrm{s}}^{3}\right)+O\left(\epsilon^{3}\right),
$$

where

$$
\begin{gathered}
\epsilon=\left|\kappa-\kappa_{\mathrm{C}}\right|^{1 / 2}, \\
s=\operatorname{sign}\left(\kappa-\kappa_{\mathrm{C}}\right), \\
\delta f_{\mathrm{s}}=\epsilon \delta \bar{f}_{\mathrm{s}} .
\end{gathered}
$$

The constants $c_{i}$ (with $i=0 \ldots 3$ ) are given by Eqs. (A11)-(A14) in terms of components of the evolution operators $\mathbf{G}^{(i)}$ and $\mathbf{H}^{(i)}$. A discussion of the higher-order terms in the slow-mode evolution equation (21) is presented in appendix A 2.

\section{B Evolution of the slow mode}

\section{Stationary solutions}

According to our assumptions, a stable stationary drop shape exists for $\kappa>\kappa_{\mathrm{C}}$ (i.e., $s=1$ ), which yields a constraint

$$
c_{0} / c_{2}>0
$$

for the coefficients in Eq. (21). We also choose the sign of the basis vector $\mathbf{g}_{1}$ to set

$$
c_{0}>0 .
$$

Equation (21) has two stationary solutions $\delta \bar{f}_{\mathrm{s}}=h_{ \pm}$ that are non-singular for $\epsilon \rightarrow 0$,

$$
h_{ \pm}= \pm s^{1 / 2} h_{0}+\epsilon h_{1}+O\left(\epsilon^{2}\right)
$$

where

$$
h_{0}=\left(\frac{c_{0}}{c_{2}}\right)^{1 / 2}
$$

$$
h_{1}=-\frac{1}{2} s\left(\frac{c_{1}}{c_{2}}+\frac{c_{3}}{c_{2}} h_{0}^{2}\right) .
$$

For supercritical values of the capillary parameter $(s=1)$, the solutions (27) are real. As shown in the following subsection, $h_{-}$is stable and $h_{+}$is unstable. For $\kappa \rightarrow \kappa_{\mathrm{C}}$ the two stationary drop shapes merge according to (24). For $s=-1$, the nonsingular stationary solutions are complex and thus, unphysical.

\section{Time-dependent behavior}

Integration of Eq. (21) yields implicit relations for the time-evolution of the slow mode. The result, accurate to $O(\epsilon)$, is

$$
\epsilon t^{\prime}=\tau_{0} \ln \frac{\left|\delta \bar{f}_{\mathrm{s}}-h_{+}\right|^{g_{+}(\epsilon)}}{\left|\delta \bar{f}_{\mathrm{s}}-h_{-}\right|^{g_{-}(\epsilon)}}
$$

for $s=1$, and

$$
\epsilon t^{\prime}=2 \tau_{0} \arctan \left[h_{0}^{-1}\left(\delta \bar{f}_{\mathrm{s}}-\epsilon h_{1}\right)\right]-q \tau_{0} \ln \left(\delta \bar{f}_{\mathrm{s}}^{\prime 2}+h_{0}^{2}\right)
$$

for $s=-1$, where

$$
t^{\prime}=t-t_{0},
$$

is the time shifted by the integration constant $t_{0}$,

$$
\tau_{0}=\frac{1}{2}\left(c_{0} c_{2}\right)^{-1 / 2}
$$

and

$$
g_{ \pm}(\epsilon)=1 \mp q \epsilon
$$


with

$$
q=c_{0}^{1 / 2} c_{2}^{-3 / 2} c_{3} .
$$

For small perturbations from the stationary states, Eq. (30) implies

$$
\delta \bar{f}_{\mathrm{s}}=h_{ \pm}+A_{0} \exp \left( \pm t^{\prime} / \tau_{ \pm}\right),
$$

where $A_{0}$ is the amplitude of the perturbation, and the time scales are given by

$$
\tau_{ \pm}=\epsilon^{-1} g_{ \pm}(\epsilon) \tau_{0} .
$$

Equation (36) indicates that $h_{-}$is stable and $h_{+}$is unstable. Drop evolution is slow in the near-critical regime, because the time scales (37) diverge for $\epsilon \rightarrow 0$.

Relations (30) and (31) can be inverted; here we present only the leading-order results. For supercritical values of the capillary parameter $\left(\kappa>\kappa_{\mathrm{C}}\right)$ Eq. (30) yields

$$
\begin{aligned}
\delta \bar{f}_{\mathrm{s}}=-h_{0} \operatorname{coth}\left(\epsilon t^{\prime} / \tau_{0}\right), & \left|\delta \bar{f}_{\mathrm{s}}\right|>h_{0} ; \\
\delta \bar{f}_{\mathrm{s}}=-h_{0} \tanh \left(\epsilon t^{\prime} / \tau_{0}\right), & -h_{0}<\delta \bar{f}_{\mathrm{s}}<h_{0} .
\end{aligned}
$$

The solution (38) has two branches: the branch $t^{\prime}>0$ (where $\delta \bar{f}_{\mathrm{s}}<-h_{0}$ ) corresponds to trajectory $l_{1}$ in Fig. 1, whereas the branch $t^{\prime}<0$ (where $\delta \bar{f}_{\mathrm{s}}>-h_{0}$ ) corresponds to trajectory $l_{3}$. The solution (39) has one branch, which corresponds to the trajectory $l_{2}$. For subcritical values of the capillary parameter $\left(\kappa<\kappa_{\mathrm{C}}\right)$, Eq. (31) yields

$$
\delta \bar{f}_{\mathrm{s}}=h_{0} \tan \left(\epsilon t^{\prime} / \tau_{0}\right),
$$

which has the asymptotic behavior $\delta \bar{f}_{\mathrm{s}} \rightarrow \pm \infty$ for $\epsilon t^{\prime} / \tau_{0} \rightarrow \pm \frac{1}{2} \pi$.

\section{Parameter choice}

To characterize near-critical drop behavior in computer simulations or in an experiment, an appropriate measurement of the slow-mode amplitude is needed. However, the explicit form of the slow mode is usually unknown or difficult to obtain.

The slow mode decomposition (13)-(20) can be performed analytically for nearly-spherical critical drop shapes (e.g., high viscosity drops in near-straining flows ${ }^{16}$ ), and explicit expressions for the coefficients $c_{i}$ can be obtained in this case. For higher drop deformations, the linear-perturbation problem (15)-(17) can be solved numerically, but evaluation of higher-order terms would be difficult. A direct experimental measurement of the form of the slow mode is infeasible.

An explicit determination of the slow mode is, however, unnecessary: the critical drop behavior can be observed using any shape parameter

$$
l=l(\mathbf{f})
$$

that has a sufficiently strong dependence on $\delta \mathbf{f}_{\mathrm{s}}$.

To characterize the critical behavior of a parameter $l$, we expand it around the critical shape $\mathbf{f}_{\mathrm{C}}$. The fast modes $\delta \mathbf{f}_{\mathrm{f}}$ are then eliminated using quasistatic relation (A10). The resulting expansion of $l$ is

$$
l=l_{\mathrm{C}}+\epsilon \delta \bar{l}
$$

with

$$
\delta \bar{l}=a_{1} \delta \bar{f}_{\mathrm{s}}+\epsilon\left(s a_{0}+a_{2} \delta \bar{f}_{\mathrm{s}}^{2}\right)+O\left(\epsilon^{2}\right),
$$

where

$$
l_{\mathrm{C}}=l\left(\mathbf{f}_{\mathrm{C}}\right),
$$

and $a_{1}>0$ is assumed. Using relation (43) to eliminate $\delta \bar{f}_{\mathrm{s}}$ from Eq. (21), the evolution equation for the shape parameter $\delta \bar{l}$ is obtained,

$$
\frac{\mathrm{d}}{\mathrm{d} t} \delta \bar{l}=\epsilon\left(-s b_{0}+b_{2} \delta \bar{l}^{2}\right)+\epsilon^{2}\left(s b_{1} \delta \bar{l}+b_{3} \delta \bar{l}^{3}\right)+O\left(\epsilon^{3}\right) .
$$

This equation has the same form as the slow-mode evolution equation (21), except for different values of the expansion coefficients. Accordingly, the stationary and time-dependent solutions derived in Sec. IV B apply to $\delta \bar{l}$, after the substitution of the coefficients $b_{i}$ for $c_{i}$.

In this paper, critical drop behavior is shown using the drop length (normalized by $2 a$ ) as the parameter $l$. Drop length exhibits strong dependence on the critical mode, and is convenient to monitor. With an appropriate choice of the shape parameter $l$, the values of the coefficients $b_{i}$ can be extracted from experimental or numerical data without a prior knowledge of the exact form of the slow mode.

\section{NUMERICAL RESULTS}

Our phenomenological theory has been tested using results of numerical simulations. In Sec. V A, a detailed analysis is presented for a drop with $\eta=1$, in axisymmetric straining flow. An example of near-critical drop behavior in shear flow is given in Sec. VB.

\section{A Axisymmetric linear flow}

Numerical results discussed in Sec. III B show that the essential features of our system are consistent with predictions based on the assumption of saddle-node bifurcation at $\kappa=\kappa_{\mathrm{C}}$. Accordingly, after the fast modes are relaxed, drop evolution is fully characterized by the amplitude of a single slow mode. The critical capillary number corresponds to $i=0$ at the minimum of the phase-space relation $i=i(l)$. For $\kappa>\kappa_{\mathrm{C}}$, there is one stable and one unstable stationary state.

In the remaining part of the present section, we present detailed quantitative tests of the theory. 


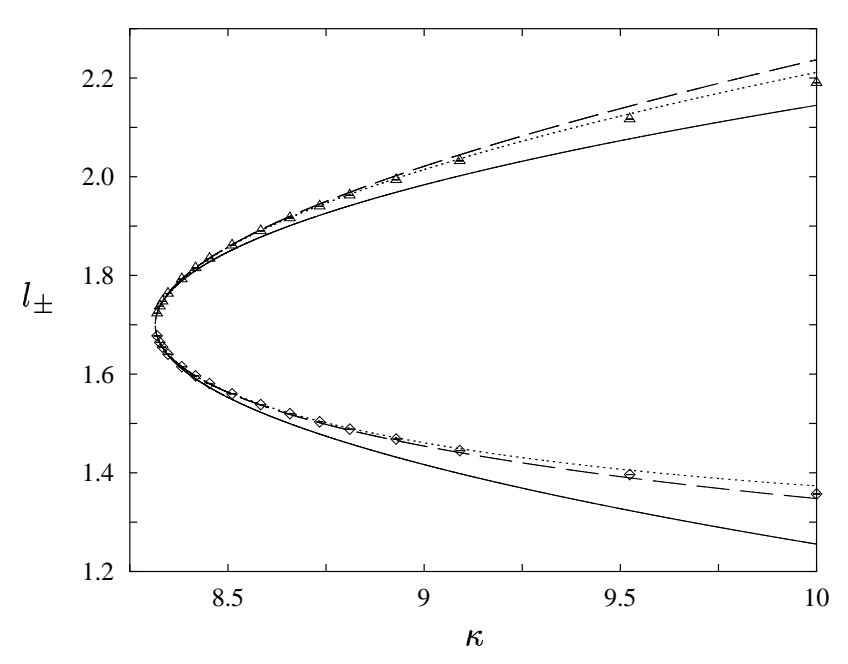

FIG. 4: Stationary drop length versus capillary parameter. Numerical results for stable length $l_{-}$(diamonds), unstable length $l_{+}$(triangles). Expansion in powers of $\epsilon$, with expansion coefficients corresponding to solid lines in Fig. 5, truncated at $O(\epsilon)$ (solid line), at $O\left(\epsilon^{2}\right)$ (dashed line), at $O\left(\epsilon^{3}\right)$ (dotted line).

\section{Drop behavior near stationary states}

We first consider the stable and unstable stationary drop lengths $l_{-}$and $l_{+}$. In Fig. 4, the stationary lengths are shown unscaled versus the capillary parameter, and in Fig. 5 in a rescaled form. As discussed in appendix A 2, the rescaled stationary length difference

$$
d=\frac{1}{2} \epsilon^{-1}\left(l_{+}-l_{-}\right),
$$

and the average length

$$
L=\frac{1}{2}\left(l_{+}+l_{-}\right)
$$

are regular functions of $\kappa$. Numerical results shown in Fig. 5 are consistent with this behavior. The linear asymptotic form of $d$, as plotted in Fig. 5(a), corresponds to

$$
\kappa_{\mathrm{C}}=8.315 .
$$

The lines in Figs. 5( $a)$ and 5(b) represent the asymptotic behavior

$$
d=l_{1}+l_{3}\left(\kappa-\kappa_{\mathrm{C}}\right), \quad L=l_{0}+l_{2}\left(\kappa-\kappa_{\mathrm{C}}\right),
$$

where the values

$$
l_{0}=1.700
$$

for the critical length, and $l_{1}=0.3426, l_{2}=0.0548$, $l_{3}=-0.0117$ are obtained by matching to the numerical data. The results (48) and (50) are consistent with the values reported by Navot $^{12}$. The approximations up to
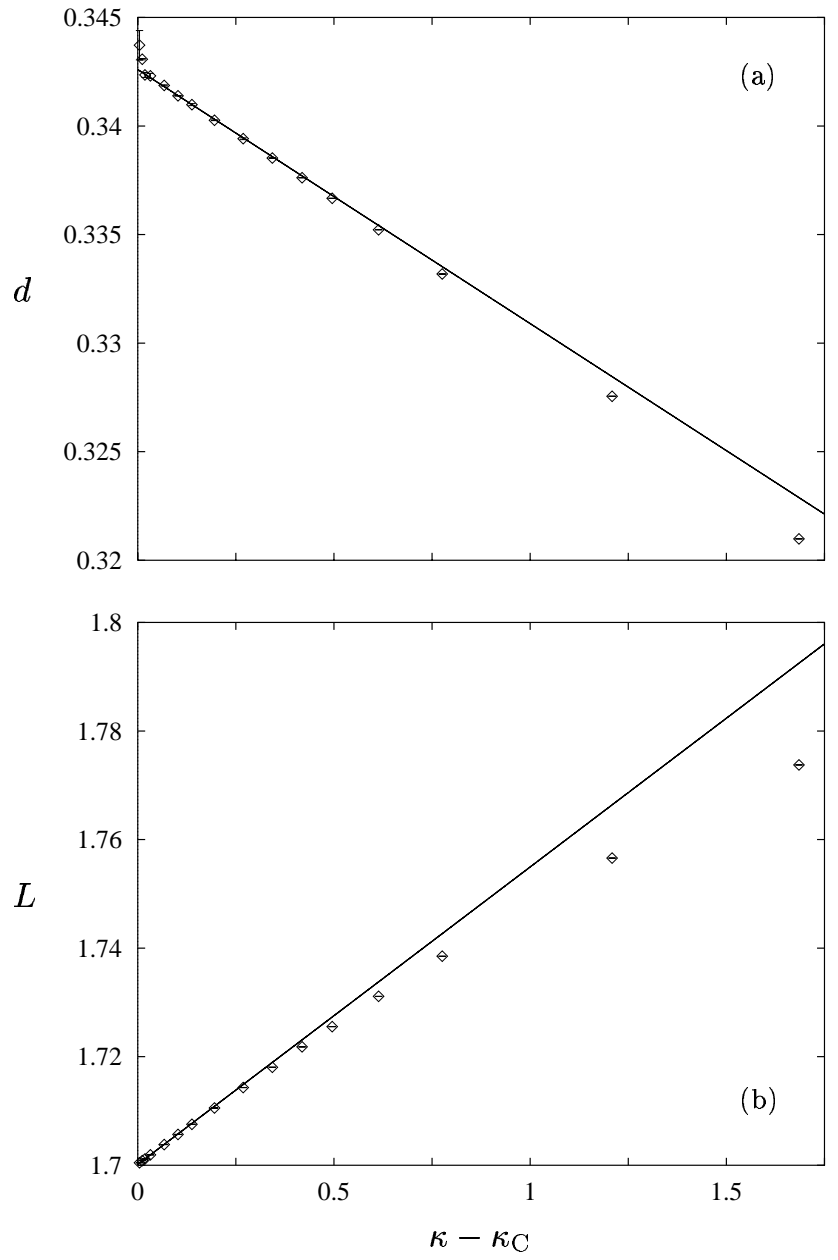

FIG. 5: Rescaled drop length difference (a), and average drop-length (b), versus $\kappa-\kappa_{\mathrm{C}}$. Numerical results (diamonds); asymptotic behavior (49) (solid lines).

$O\left(\epsilon^{3}\right)$ for $l_{-}$and $l_{+}$, with the coefficients given above, are shown in Fig. (4).

Next we consider the inverse time scales

$$
\alpha_{ \pm}= \pm \tau_{ \pm}^{-1},
$$

characterizing the slow-mode behavior (36) near the stable and unstable stationary states. These time scales, obtained from an analysis of the exponential evolution of the drop length for $l \approx l_{ \pm}$, are shown in Fig. 6 in an unscaled, and in Fig. 7 in a rescaled form.

The results indicate that the behavior of the time scales in the near-critical regime is analogous to the behavior of the stationary drop lengths. As discussed in appendix A 2, the rescaled quantities

$$
\delta=\frac{1}{2} \epsilon^{-1}\left(\alpha_{+}-\alpha_{-}\right)
$$

and

$$
A=\frac{1}{2}\left(\alpha_{+}+\alpha_{-}\right)
$$




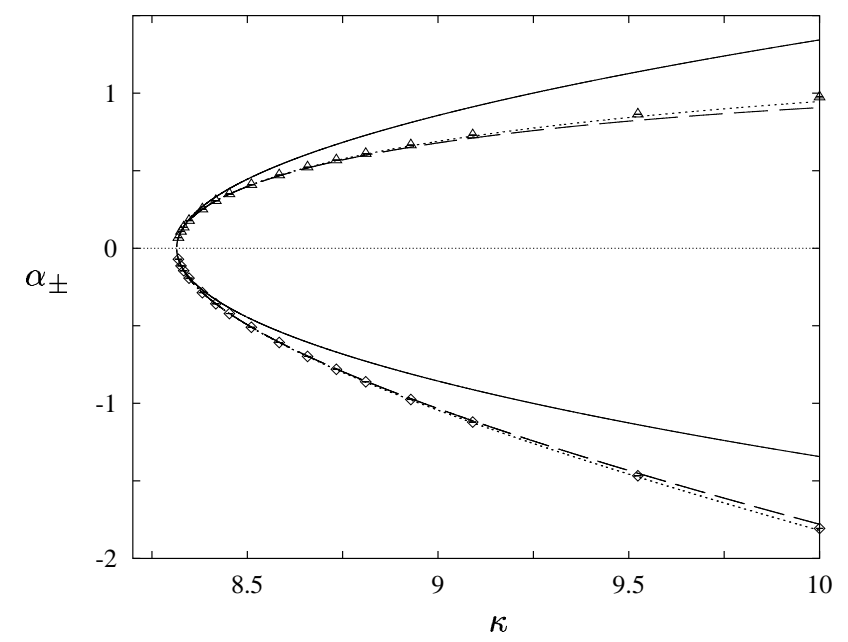

FIG. 6: Inverse time scale (51) for the evolution near the stable and unstable stationary states. The meaning of symbols as in Fig. 4, except that the results are for $\alpha_{ \pm}$, and the expansion coefficients correspond to the lines in Fig. 7.

are regular in $\kappa$; the results shown in Fig. 7 are consistent with this prediction. The rescaled time-scale difference (52) shown in Fig. 7( $a$ ) corresponds to the critical value (48) of the capillary parameter, consistently with the stationary results. The lines in Figs. 7( $a)$ and $7(b)$ represent the asymptotic behavior

$$
\delta=\alpha_{1}+\alpha_{3}\left(\kappa-\kappa_{\mathrm{C}}\right), \quad A=\alpha_{2}\left(\kappa-\kappa_{\mathrm{C}}\right),
$$

where $\alpha_{1}=1.035, \alpha_{2}=-0.259$, and $\alpha_{3}=0.018$. (The value reported by Navot $^{12}$ for the coefficient corresponding to $\alpha_{1}$ is $20 \%$ smaller.)

The accuracy of the data presented in Figs. 4-7 has been estimated by comparing the values of $l_{ \pm}$and $\alpha_{ \pm}$ obtained from an analysis of the drop length history just below and just above the stable and unstable stationary states. The evolution in the regimes $l<l_{ \pm}$and $l>l_{ \pm}$ corresponds to different initial conditions, and the associated results for $l_{ \pm}$and $\alpha_{ \pm}$slightly differ because of numerical inaccuracies. In Figs. 4-7, the difference is represented in the form of error bars, which in most cases are invisible on the scale of the figure. However, the error bars occasionally become larger, particularly for the rescaled time scale $\delta$ shown in Fig. 7(a), where the inaccuracies are magnified by the factor $\epsilon^{-1}$ in the definition (52).

\section{Drop evolution}

The theoretical predictions given in Sec. IV B 2 will now be compared to our numerical results for the droplength evolution.

For $\kappa>\kappa_{\mathrm{C}}$, we focus on the non-exponential evolution described by relation (30) (where the slow-mode parame-
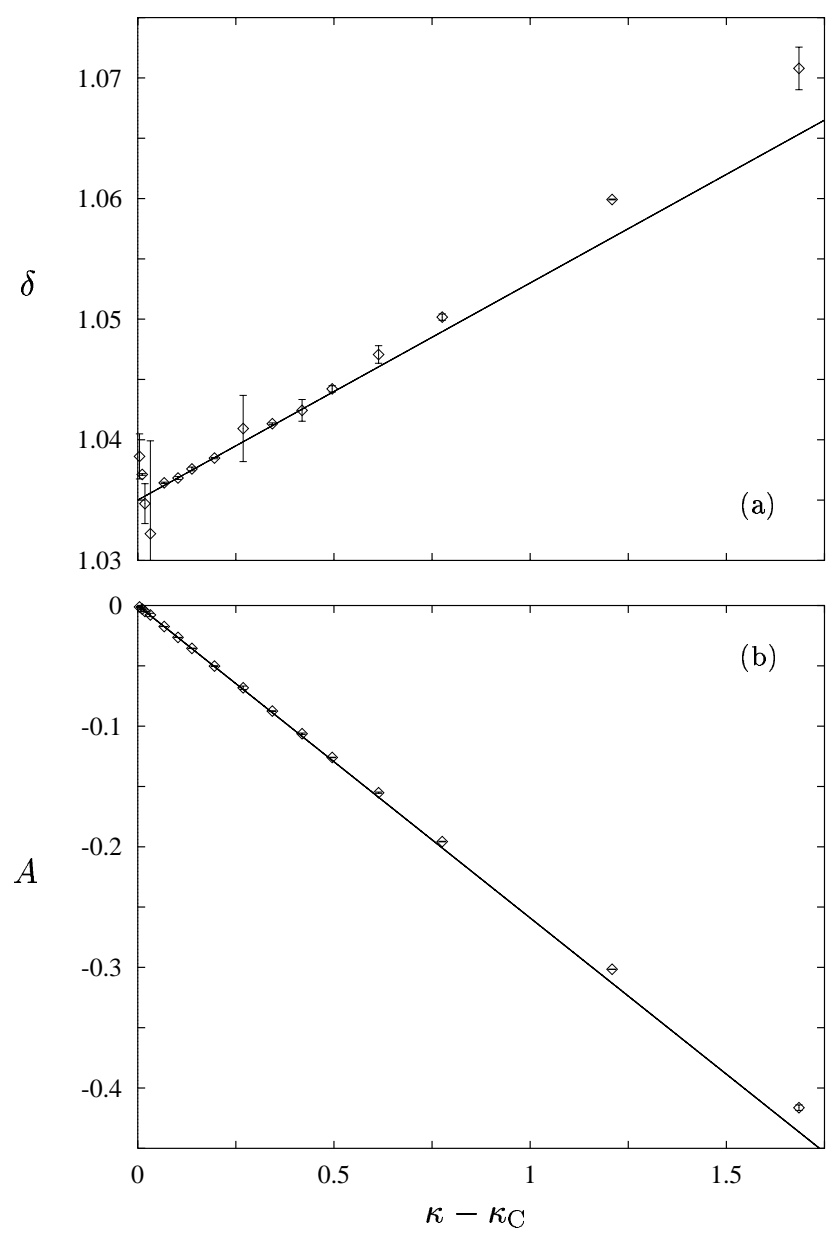

FIG. 7: Rescaled inverse time scale difference (a), and average inverse time scale $(b)$, versus $\kappa-\kappa_{\mathrm{C}}$. Numerical results (diamonds); asymptotic behavior (54) (solid lines).

ter $\delta f_{\mathrm{s}}$ is represented by the drop length $l$, as before). As discussed in the preceding subsection, detailed estimates for the accuracy of the expansions of $l_{ \pm}$and $\tau_{ \pm}$in powers of $\epsilon$ are provided by the results shown in Figs. 4-7. Therefore, to verify other aspects of our approximations, we use in Eq. (30) the exact values of the stationary lengths and the time scales (37). The comparison between Eq. (30) and numerical results for drop evolution in the regime $\kappa>\kappa_{\mathrm{C}}$ is presented in Figs. 8 and 9.

The results for $l<l_{-}$are shown in Fig. $8(a)$, where $\ln \left(l_{-}-l\right)$ is plotted as a function of the rescaled time $\epsilon t^{\prime} / \tau_{0}$. The time is shifted so that Eq. (30) and the numerical values agree for large $t^{\prime}$. The left ends of the curves representing the simulation correspond to the initial spherical drop shape. The theoretical curves are truncated at $t=4 \tau_{\sigma}$, where $\tau_{\sigma}=\mu a / \sigma$ is the capillary relaxation time. Thus, the simulations and theory are compared only in the long-time regime.

The results for $l>l_{+}$are shown in Fig. $8(b)$, where $\ln \left(l-l_{+}\right)$is plotted as a function of the rescaled time. 

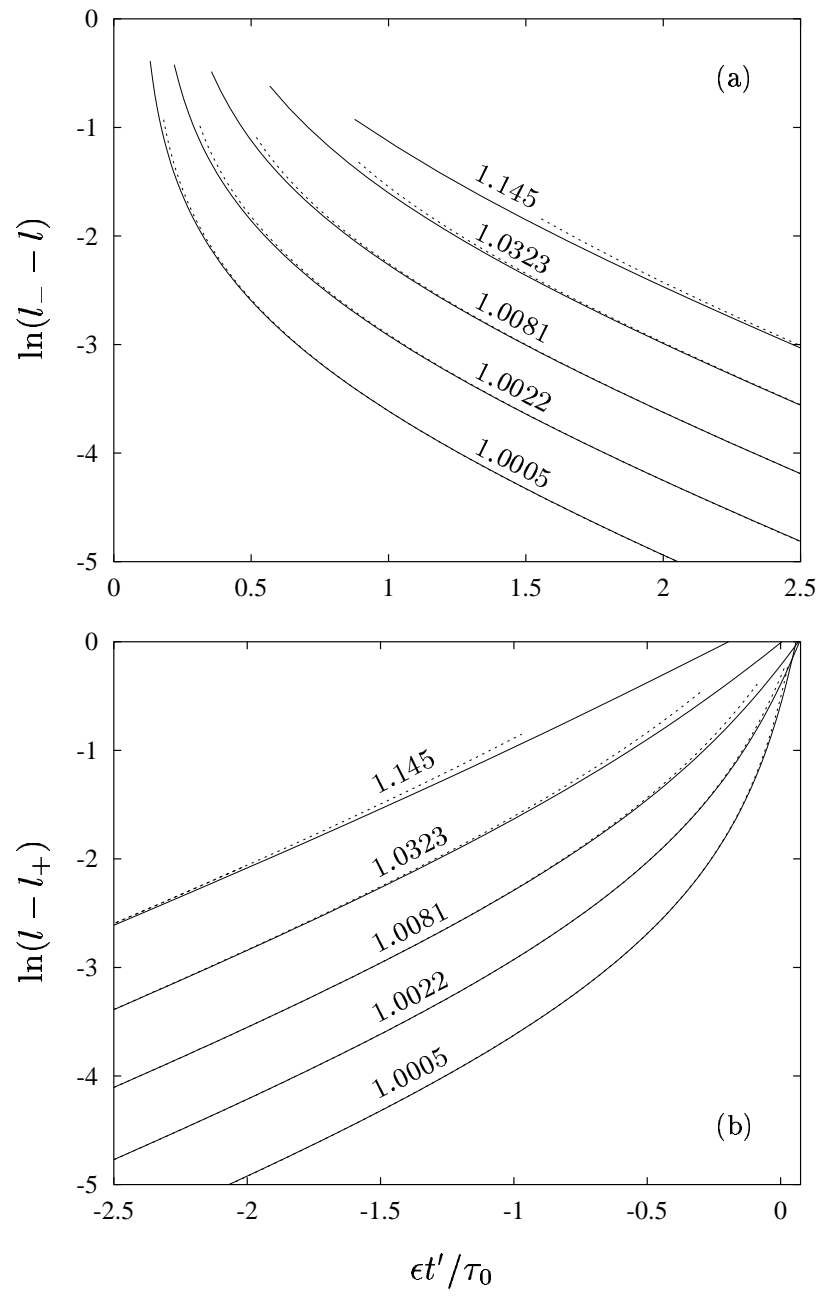

FIG. 8: Drop length versus rescaled time for $l<l_{-}(a)$, and $l>l_{+}(b)$. Numerical results (solid lines); expression (30) (dotted lines). Values of $\kappa / \kappa_{\mathrm{C}}$ as labeled.

The time is shifted so that Eq. (30) and the numerical values agree for large negative values of $t^{\prime}$. For $t^{\prime} \rightarrow 0$, the theoretical curves are truncated for $l / l_{0}>1.5$.

The linear behavior for $t \gg 0$ in Fig. $8(a)$, and for $t \ll 0$ in Fig. $8(b)$, corresponds to the exponential evolution near the stable and unstable stationary states. By construction, within the numerical accuracy, approximation (30) and the numerical results agree in these regimes. The results corresponding to nonlinear portions of the curves indicate that the approximation (30) is valid also in the regions where the evolution of the system is nonexponential, as long as fast modes have relaxed, and the perturbation from the critical state is sufficiently small.

Figure 9 presents drop evolution for $l_{-}<l<l_{+}$. In this case, a transition between two exponential asymptotic behaviors occurs; accordingly, the plot of $\ln \left[\left(l_{+}-l\right) /\left(l-l_{-}\right)\right]$is shown. The time is shifted so that the numerical and theoretical curves match at $t^{\prime}=0$. The results indicate, that the approximation (30) is quite accurate up to moderate values of $\kappa-\kappa_{\mathrm{C}}$.

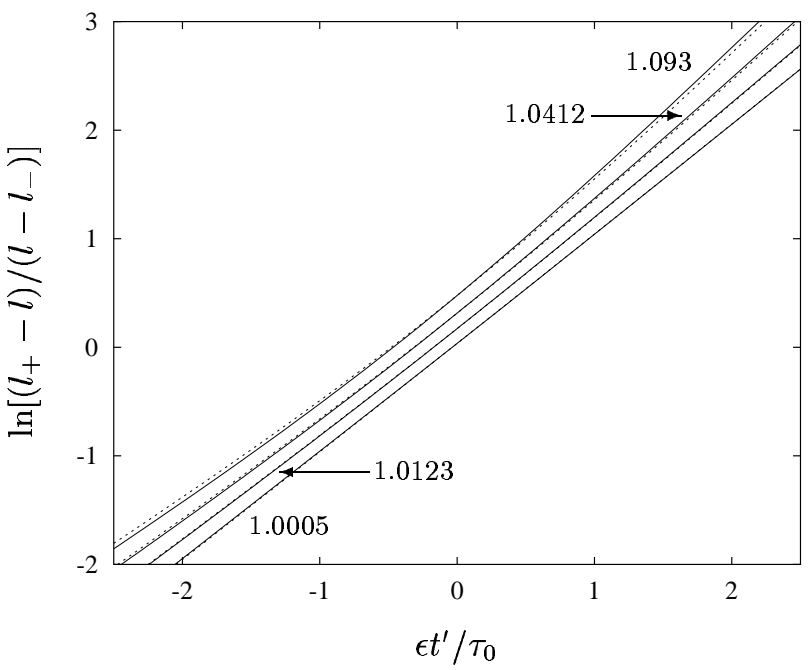

FIG. 9: Same as Fig. 8, except that $l_{-}<l<l_{+}$.

For $\kappa<\kappa_{\mathrm{C}}$, there are no stationary drop shapes. In this regime, numerical results for drop length are compared to the leading-order solution (40) and the firstorder solution (31), with parameter values obtained from the expansions (49) and (54). The plot of drop length versus the rescaled time is shown in Fig. 10 for several values of the capillary parameter. The results are consistent with our theory.

\section{B Shear flow}

Using our recently developed boundary-integral algorithm ${ }^{17}$, we performed three-dimensional simulations of drop evolution in linear flows with non-vanishing rotational component. In all cases considered, the instability of stationary drop shapes in the near-critical regime $\kappa \approx \kappa_{\mathrm{C}}<\infty$ occurs through the mechanism described in Sec. IV.

In Fig. 11 we present results for a drop with $\eta=1$ in shear flow

$$
\mathbf{v}_{0}=\dot{\gamma} y \mathbf{e}_{x} .
$$

In the regime $\kappa>\kappa_{\mathrm{C}}$, the stable stationary drop length is plotted as

$$
\Delta l^{2}=\left(l_{-}-l_{0}\right)^{2}
$$

versus $\kappa$. The value for the critical length, $l_{0}=2.35$, was obtained by matching the numerical results to the linear asymptotic behavior

$$
\Delta l^{2}=l_{1}^{2}\left(\kappa-\kappa_{\mathrm{C}}\right)+O\left(\kappa-\kappa_{\mathrm{C}}\right)^{3 / 2}
$$

[which follows from Eqs. (46), (47), and (49)]. In the regime $\kappa<\kappa_{\mathrm{C}}$, the minimal elongation rate along a drop trajectory,

$$
i_{\min }=\min (d l / d t)
$$




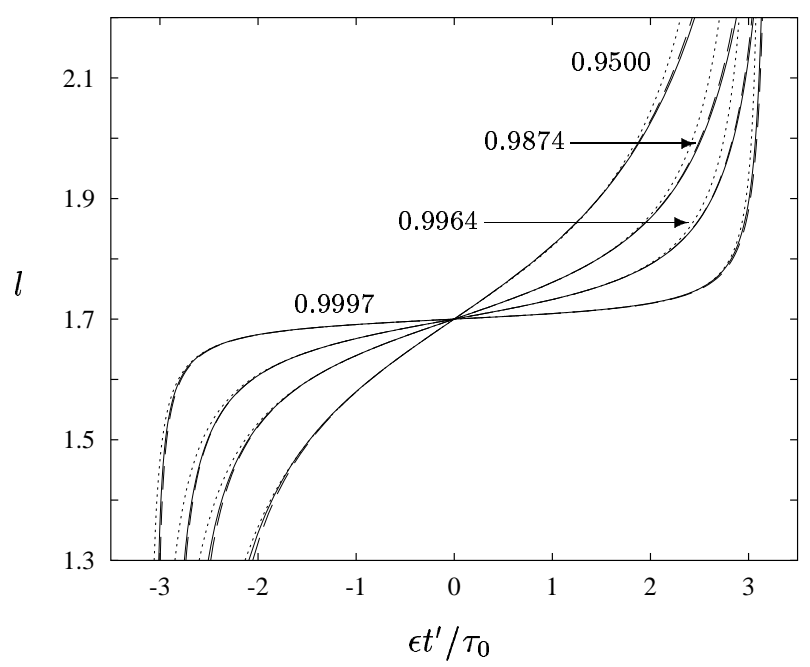

FIG. 10: Drop length versus rescaled time. Numerical results (solid line); leading-order approximation (dotted line); firstorder approximation (dashed line). Values of $\kappa / \kappa_{\mathrm{C}}$ as labeled.

is shown. According to Eq. (45),

$$
i_{\min }=b_{0}\left(\kappa-\kappa_{\mathrm{C}}\right)+O\left(\kappa-\kappa_{\mathrm{C}}\right)^{2}
$$

is a regular function of $\kappa$. The results shown in Fig. 11 illustrate this behavior.

Within numerical accuracy, the two lines representing the asymptotic behaviors (57) and (59) in Fig. 11 correspond to the same value of the critical capillary parameter. Extrapolation of our numerical results with the number of boundary elements used in the calculations yields

$$
\kappa_{\mathrm{C}}=2.320 \text {. }
$$

The asymptotic formulas (57) and (59) can be efficiently used to determine critical capillary numbers from numerical or experimental data.

A detailed analysis of near-critical drop behavior in linear flows with rotation will be reported in a forthcoming paper. ${ }^{19}$

\section{CONCLUSIONS}

Our study provides a phenomenological framework for describing the dynamics of drops in creeping flows under near-critical conditions. We have shown that in the near-critical regime drop behavior is dominated by the evolution of a single slow-mode, and at the critical point the system undergoes a saddle-node bifurcation. Thus, the transient dynamics and stationary states are characterized by a critical exponent with the classical value of $\frac{1}{2}$ (in analogy to the Landau theory of phase transitions).

In linear flows with nonzero vorticity a stabilizing mechanism, alternative to capillary forces, is provided

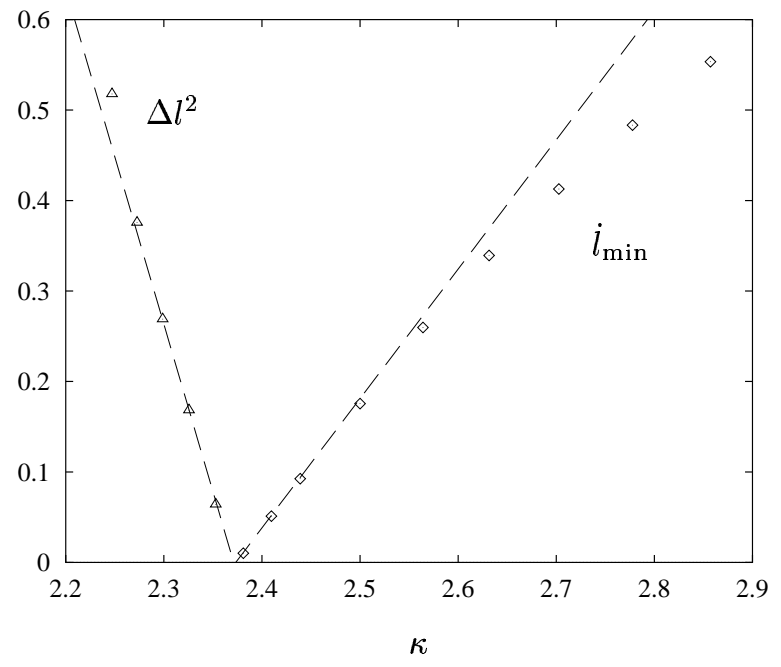

FIG. 11: Stable stationary drop length difference $\Delta l^{2}$ (diamonds), and minimum slope $i$ (triangles) versus capillary parameter $\kappa$, from boundary-integral simulations for shear flow, $\eta=1$. The critical capillary parameter $\kappa_{\mathrm{C}}$ corresponds to the intersection of the dashed lines and the horizontal axis.

by drop rotation ${ }^{15,20}$. As a result, in such flows there exists a critical viscosity ratio $\lambda_{\mathrm{C}}$ beyond which stationary states occur even without surface tension. An extension of our formalism to describe the near-critical behavior of drops for $\lambda \approx \lambda_{\mathrm{C}}$, as well as the dependence of $\lambda_{\mathrm{C}}$ on the flow type, will be presented in the forthcoming publications $^{16,19}$.

In Ref. 16, multiple stable stationary states are shown to occur for high-viscosity drops in two-dimensional flows with a small vorticity component. Under these conditions, there exists a branch of stationary solutions that is limited at both ends, $\kappa_{\mathrm{C}}>\kappa>\kappa_{\mathrm{C}}^{\prime}$, in contrast to Eq. (8). With minor modifications, the theory developed herein applies to both near-critical regions $\kappa \approx \kappa_{\mathrm{C}}, \kappa_{\mathrm{C}}^{\prime}$.

An important application of our theory is in the accurate determination of critical parameter values from numerical simulations or experiments in a near-critical regime. Numerical results for the critical capillary number in two-dimensional linear flows will be presented in the forthcoming paper ${ }^{19}$.

Our approach may also be useful in analyzing the dynamics of polymer molecules undergoing coil-stretch transitions in external flows ${ }^{21,22}$. These transitions result from the interplay between entropically-driven relaxation and convection by an imposed flow ${ }^{23}$. The essential mechanism is, thus, similar to the one studied herein. 


\section{APPENDIX A: EVOLUTION EQUATION FOR THE SLOW MODE}

1 Derivation of Eq. (21)

Two coupled evolution equations for the slow and the fast modes are derived by applying to both sides of Eq. (13) projection operators $\hat{\mathbf{P}}_{\mathrm{s}}$ and $\hat{\mathbf{P}}_{\mathrm{f}}$ onto subspaces (19) and $(20)$,

$\frac{\mathrm{d}}{\mathrm{d} t} \delta \mathbf{f}_{\mathrm{s}}=-\delta \kappa \mathbf{H}_{\mathrm{s}}-\delta \kappa \mathbf{H}_{\mathrm{s}}^{(1)} \cdot \delta \mathbf{f}+\frac{1}{2}\left(\mathbf{B}_{\mathrm{s}}^{(2)}-\delta \kappa \mathbf{H}_{\mathrm{s}}^{(2)}\right): \delta \mathbf{f} \delta \mathbf{f}+\ldots$,

$$
\begin{aligned}
\frac{\mathrm{d}}{\mathrm{d} t} \delta \mathbf{f}_{\mathrm{f}}= & -\delta \kappa \mathbf{H}_{\mathrm{f}}+\mathbf{B}_{\mathrm{f}}^{(1)} \cdot \delta \mathbf{f}_{\mathrm{f}}-\delta \kappa \mathbf{H}_{\mathrm{f}}^{(1)} \cdot \delta \mathbf{f} \\
& +\frac{1}{2}\left(\mathbf{B}_{\mathrm{f}}^{(2)}-\delta \kappa \mathbf{H}_{\mathrm{f}}^{(2)}\right): \delta \mathbf{f} \delta \mathbf{f}+\ldots,
\end{aligned}
$$

where $\delta \kappa=\kappa-\kappa_{\mathrm{C}}$,

$$
\mathbf{B}^{(i)}=\mathbf{G}^{(i)}-\kappa_{\mathrm{C}} \mathbf{H}^{(i)},
$$

and

$$
\mathbf{A}_{a}=\hat{\mathbf{P}}_{a} \mathbf{A}, \quad a=\mathrm{s}, \mathrm{f},
$$

(with $\mathbf{A}=\mathbf{f}, \mathbf{H}, \mathbf{B}, \ldots$ ). The operators $\mathbf{G}, \mathbf{H}, \mathbf{B}$ are evaluated at $\mathbf{f}=\mathbf{f}_{\mathrm{C}}$. Equations (A1) and (A2) were simplified using relation (14), and the identities

$$
\mathbf{B}_{\mathrm{s}}^{(1)} \mathbf{f}=0, \quad \mathbf{B}_{\mathrm{f}}^{(1)} \mathbf{f}_{\mathrm{s}}=0 .
$$

These identities follow from (15) and the observation that the subspaces $\mathbf{f}_{\mathrm{s}}$ and $\mathbf{f}_{\mathrm{f}}$ correspond to different eigenvalues of the operator $\mathbf{B}^{(1)}$.

Equation (A2) indicates that, at the leading order in $\delta \kappa$, the evolution of the fast mode near the stationary state is governed by the constant term $-\delta \kappa \mathbf{H}_{\mathrm{f}}$ and the linear term $\mathbf{B}_{\mathrm{f}}^{(1)} \cdot \delta \mathbf{f}_{\mathrm{f}}$. In contrast, the linear term is missing from Eq. (A1) at the leading order; thus, the evolution of the slow mode is governed by the constant term and the quadratic term in $\delta \mathbf{f}_{\mathrm{s}}$. Accordingly, the following scalings are appropriate for the slow and the fast modes

$$
\begin{array}{r}
\delta \mathbf{f}_{\mathrm{s}}=\epsilon \delta \overline{\mathbf{f}}_{\mathrm{s}}, \\
\delta \mathbf{f}_{\mathrm{f}}=\epsilon^{2} \delta \overline{\mathbf{f}}_{\mathrm{f}},
\end{array}
$$

where $\epsilon$ is given by Eq. (22). In the rescaled variables, the leading-order terms in the evolution equations for the slow and the fast modes are

$$
\begin{aligned}
\frac{\mathrm{d}}{\mathrm{d} t} \delta \overline{\mathbf{f}}_{\mathrm{s}}= & \epsilon\left(-s \mathbf{H}_{\mathrm{s}}+\frac{1}{2} \mathbf{B}_{\mathrm{s}}^{(2)}: \delta \overline{\mathbf{f}}_{\mathrm{s}} \delta \overline{\mathbf{f}}_{\mathrm{s}}\right) \\
& +\epsilon^{2}\left(-s \mathbf{H}_{\mathrm{s}}^{(1)} \cdot \delta \overline{\mathbf{f}}_{\mathrm{s}} \mathbf{B}_{\mathrm{s}}^{(2)}: \delta \overline{\mathbf{f}}_{\mathrm{f}} \delta \overline{\mathbf{f}}_{\mathrm{s}}\right. \\
& \left.+\frac{1}{6} \mathbf{B}_{\mathrm{s}}^{(3)}: \delta \overline{\mathbf{f}}_{\mathrm{s}} \delta \overline{\mathbf{f}}_{\mathrm{s}} \delta \overline{\mathbf{f}}_{\mathrm{s}}\right)+O\left(\epsilon^{3}\right) \\
\frac{\mathrm{d}}{\mathrm{d} t} \delta \overline{\mathbf{f}}_{\mathrm{f}}=-s \mathbf{H}_{\mathrm{f}}+ & \mathbf{B}_{\mathrm{f}}^{(1)} \cdot \delta \overline{\mathbf{f}}_{\mathrm{f}}+\frac{1}{2} \mathbf{B}_{\mathrm{f}}^{(2)}: \delta \overline{\mathbf{f}}_{\mathrm{s}} \delta \overline{\mathbf{f}}_{\mathrm{s}}+O(\epsilon),
\end{aligned}
$$

where $s$ is defined by (23).

Equation (A8) indicates that the evolution of the slow mode occurs on the time scale that diverges at $\kappa \rightarrow \kappa_{\mathrm{C}}$ with the critical exponent $\frac{1}{2}$. At the leading order, equation for the slow mode is independent of the fast modes, but at the order $O\left(\epsilon^{2}\right)$, the slow and fast modes are coupled. However, on the time scale $t=O(1)$, fast modes relax towards the quasi-stationary value

$$
\delta \overline{\mathbf{f}}_{\mathrm{f}}=\mathbf{B}_{\mathrm{f}}^{(1)^{-1}} \cdot\left(s \mathbf{H}_{\mathrm{f}}-\frac{1}{2} \mathbf{B}_{\mathrm{f}}^{(2)}: \delta \overline{\mathbf{f}}_{\mathrm{s}} \delta \overline{\mathbf{f}}_{\mathrm{s}}\right)+O(\epsilon),
$$

and then follow the evolution of the slow mode adiabatically. Inserting the above expression into Eq. (A8) yields the closed long-time evolution equation (21) for the slow mode, where

$$
c_{0} \mathbf{g}_{1}=\mathbf{H}_{\mathrm{s}},
$$

$$
\begin{gathered}
c_{1} \mathbf{g}_{1}=\left[-\mathbf{H}_{\mathrm{s}}^{(1)}+\mathbf{B}_{\mathrm{s}}^{(2)} \cdot\left(\mathbf{B}_{\mathrm{f}}^{(1)^{-1}} \cdot \mathbf{H}_{\mathrm{f}}\right)\right] \cdot \mathbf{g}_{1}, \\
c_{2} \mathbf{g}_{1}=\frac{1}{2} \mathbf{B}_{\mathrm{s}}^{(2)}: \mathbf{g}_{1} \mathbf{g}_{1}, \\
c_{3} \mathbf{g}_{1}=\left[\frac{1}{6} \mathbf{B}_{\mathrm{s}}^{(3)}-\frac{1}{2} \mathbf{B}_{\mathrm{s}}^{(2)} \cdot\left(\mathbf{B}_{\mathrm{f}}^{(1)}{ }^{-1} \cdot \mathbf{B}_{\mathrm{f}}^{(2)}\right)\right]: \mathbf{g}_{1} \mathbf{g}_{1} \mathbf{g}_{1} .
\end{gathered}
$$

\section{Higher-order terms}

Elimination of fast modes can be continued to an arbitrary order. This procedure yields the slow-mode evolution equation of the form

$$
\epsilon \frac{\mathrm{d}}{\mathrm{d} t} \delta \bar{f}_{\mathrm{s}}=\epsilon^{2}\left(-s c_{0}+c_{2} \delta \bar{f}_{\mathrm{s}}^{2}\right)+\epsilon^{3} \psi\left(\delta \bar{f}_{\mathrm{s}} ; \epsilon\right),
$$

with $\psi$ given as a series in $\delta \bar{f}_{\mathrm{s}}$ and $\epsilon$.

The unscaled evolution equations (A1)-(A2) are regular in $\kappa$ and $\delta f_{\mathrm{s}}$. The odd powers of $\epsilon$ in Eq. (A15) result entirely from the rescaling of the slow-mode amplitude (A6). As a consequence, the function $\psi$ satisfies the symmetry relation

$$
\psi\left(-\delta \bar{f}_{\mathrm{s}} ;-\epsilon\right)=-\psi\left(\delta \bar{f}_{\mathrm{s}} ; \epsilon\right),
$$

since the unscaled array $\delta \mathbf{f}_{\mathrm{s}}$ is invariant under a simultaneous change of sign of $\epsilon$ and $\delta \overline{\mathbf{f}}_{\mathrm{s}}$ in Eq. (A6).

It follows that the nonsingular stationary solutions $\delta \bar{f}_{\mathrm{s}}=h_{ \pm}$of equation (A15) are related by

$$
h_{+}(\epsilon)=-h_{-}(-\epsilon) .
$$

Since $h_{ \pm}$is a regular function of $\epsilon$, Eq. (A17) implies that the linear combinations $\frac{1}{2}\left(h_{+}-h_{-}\right)$and $\frac{1}{2} \epsilon\left(h_{+}+h_{-}\right)$are regular functions of $\epsilon^{2}=\kappa-\kappa_{\mathrm{C}}$. The regular expansions of (46) and (47) in $\epsilon^{2}$ follow.

The inverse time scales (51) are obtained from

$$
\alpha_{ \pm}=2 \epsilon c_{2} h_{ \pm}+\epsilon^{2} \psi^{\prime}\left(h_{ \pm}, \epsilon\right),
$$


where the prime denotes the derivative with respect to $\delta \bar{f}_{\mathrm{s}}$. Equations (A16) and (A17), thus, imply that

$$
\alpha_{+}(\epsilon)=\alpha_{-}(-\epsilon) .
$$

The regular expansions of (52) and (53) in $\epsilon^{2}$ follow from the above relation.

\section{REFERENCES}

$\dagger$ present address: Department of Chemical Engineering and Materials Science, University of Minnesota, 421 Washington Avenue S.E., Minneapolis, MN 554550132 .

${ }^{1}$ H. A. Stone, Annu. Rev. Fluid Mech. 26, 65 (1994).

2 J. P. Grace, Chem. Eng. Commun. 14, 225 (1982).

3 B. J. Bentley and L. G. Leal, J. Fluid Mech. 167, 241 (1986).

${ }^{4}$ R. A. Bruijn, Deformation and breakup of drops in simple shear flows, Ph.D. thesis, Tech. Univ. Eindhoven (1989).

${ }^{5}$ M. R. Kennedy, C. Pozrikidis, and R. Skalak, Computer Fluids 23, 251 (1994).

${ }^{6}$ H. A. Stone and L. G. Leal, J. Fluid Mech. 198, 399 (1989).

7 M. Tjahjadi and J. M. Ottino, J. Fluid Mech. 232, 191 (1991).

${ }^{8}$ F. J. Muzzio, M. Tjahjadi, and J. M. Ottino, Phys.

Rev. Lett. 1991, 54 (1991).
9 J. M. H. Janssen and H. E. H. Meijer, J. Rheol. 37, 597 (1993).

10 D. I. Bigio, C. R. Marks, and R. V. Calabrese, Int. Polym. Proc. 13, 192 (1998).

11 C. R. Marks, Drop breakup and deformation in sudden onset strong flows, Ph.D. thesis, University of Maryland at College Park (1998).

${ }^{12}$ Y. Navot, Phys. Fluids 11, 990 (1999).

13 J. Bławzdziewicz, V. Cristini, and M. Loewenberg, Bul. Am. Phys. Soc. 43, 2066 (1998).

14 D. Barthès-Biesel and A. Acrivos, J. Fluid Mech. 61, 1 (1973).

15 J. M. Rallison, J. Fluid Mech. 98, 625 (1980).

16 J. Bławzdziewicz, V. Cristini, and M. Loewenberg (2000), in preparation.

17 V. Cristini, J. Bławzdziewicz, and M. Loewenberg, J. Comput. Phys. (2000), submitted.

18 P. G. Drazin, Nonlinear Systems (Cambridge University Press, Cambridge, 1992).

19 J. Bławzdziewicz, V. Cristini, and M. Loewenberg (2000), in preparation.

${ }^{20}$ G. I. Taylor, Proc. R. Soc. Lond. A 146, 501 (1934).

${ }^{21}$ D. E. Smith, H. P. Babcock, and S. Chu, Science 283, 17224 (1999).

${ }^{22}$ P. LeDuc, C. Haber, G. Bao, and D. Wirtz, Nature 399, 564 (1999).

${ }^{23}$ P. G. De Gennes, J. Chem. Phys. 60, 5030 (1974). 